\title{
Determination nurses' knowledge about initial drugs used during emergency management of acute myocardial infarction
}

\author{
Hassanat Elbashir Mohamed Mustafa , Badria Abd Alla Mohamed Elfaki \\ Department of Nursing Practice, Faculty of Nursing, Um Al Qura University, Khartoum, Sudan
}

Received: September 6, 2016

Accepted: November 6, 2016

Online Published: December 26, 2016

DOI: $10.5430 /$ jnep.v7n5p62

URL: http://dx.doi.org/10.5430/jnep.v7n5p62

\begin{abstract}
Objective: Acute myocardial infarction is a life threatening condition that influences the physical, psychological and social dimensions of the individual. The aim of this study was conducted to determine nurses' knowledge of initial treatment during an emergency management of patients with acute myocardial infarction.

Methods: A descriptive study was used. The standardized administered questionnaires were administered on 139 critical care nurses who are employed in five teaching hospitals at Khartoum state/Sudan. Data processed using the statistical package software (SPSS); version 19, Chi-Square test was used, $p$-value of $<.05$ considered statistically significant for the analysis.

Results: The study results revealed that the total mean knowledge scores of the studied subjects related to the initial treatment of acute myocardium infarction was found to be low than bench mark with $t \& p$ values $(t=6.87$ at $p=.000)$. Also, most of studied subjects had poor level of knowledge regarding thrombolytic agents specifically about characteristics of streptokinase, indications, contraindications and complications; even among trained nurses $(p$ values $=.000)$.

Conclusions: The studied subjects had poor levels of knowledge related to the drugs used for initial management of acute myocardial infarction specifically those related to understanding the thrombolytic agents.
\end{abstract}

Key Words: Nurses knowledge, Emergency drugs, Acute myocardial infarction, Cardiac care Units, Emergency departments

\section{INTRODUCTION}

Cardiovascular emergencies are life-threatening disorders that must be recognized immediately to avoid consequences related to morbidity and mortality. ${ }^{[1]}$ Acute myocardial infarction (AMI) is caused by complete blockage of the coronary artery due to a thrombus attach to a ruptured plaque which requires an immediate diagnosis and treatment. The underlying risk factors include; numbers of modifiable and non-modifiable risk factors. The modifiable risk factors are those which are related to unhealthy life style, and can be prevented such as hyperlipidemia, diabetes mellitus, hypertension, and tobacco use. The non-modifiable risk factors are difficult to prevent, these include male gender, race and family history of atherosclerotic arterial disease. ${ }^{[2-4]}$ Patients may present with severe chest pain, dysrhythmia, cardiopulmonary arrest and structural damage, such as mitral regurgitation, rupture of the left ventricular wall or septum..$^{[5,6]}$ According to the World Health Organization (WHO), statistical data for 2011 showed that the mortality rate related to coronary artery diseases in Sudan was 212.0 per $100,000 .{ }^{[7]}$

\footnotetext{
*Correspondence: Hassanat Elbashir Mohamed Mustafa; Email: hassanat_elbashir@yahoo.com; Address: Department of Nursing Practice, Faculty of Nursing, Um Al Qura University, Khartoum, Sudan.
} 
As a common practice, initial therapy for AMI aims at the restoration of perfusion to prevent further damage to the myocardium. ${ }^{[8]}$ Initial therapy such as Morphine, Oxygen, Nitroglycerin and Aspirin (MONA) are used for stabilizing the patient's condition, as well as thrombolytic agents which have the most benefits when it is given as soon as the blockage occurred. ${ }^{[9]}$ Trained prehospital personnel such as the emergency medical service (EMS) system can provide lifesaving interventions if the patient develops cardiac arrest. ${ }^{[10]}$ While, the Streptokinase therapy is used as a measure to dissolve a clot and reopen the arteries or veins, it cannot be used in all conditions. ${ }^{[11]}$ The nurses in the emergency rooms are expected to be knowledgeable about the characteristics of the therapy and able to make a decision related to the management of acute conditions. ${ }^{[8,12-14]}$ Nurses have proven their capability not only to reduce cardiovascular risk factors, but also to adhere to treatment guidelines and protocols, decrease hospitalization and reduce morbidity and mortality of acute conditions. ${ }^{[15]}$ The American Heart Association (AHA) and the World Health Organization recognized the key role that the nurses and other team members play in supporting the goal to reduce cardiovascular diseases and disability by $25 \%$ in 2025 . $^{[15]}$ When patient presents with chest pain to emergency departments, staff should have a high index of suspicion that their pain is of cardiac. ${ }^{[16]}$ Therefore, nurses' knowledge is a significant determinant in the successful management of acute cardiovascular conditions and prevention of unnecessary morbidity and mortality.

\section{Problem statement}

Assessment of nurses' knowledge regard uses of emergency drugs such as morphine oxygen, Nitrate, Aspirin (MONA) and thrombolytic agents to dissolve a clot; which given at initial phase of myocardial infarction attack.

\section{MATERIAls AND METHOdS}

\subsection{Research design}

This is a descriptive hospital based study design.

\subsection{Setting}

The study carried out at cardiac care units (CCU) and emergency departments, affiliated to Khartoum five public hospitals; which are largest educational hospitals at Khartoum State in Sudan.

\subsection{Sample size}

There are a total coverage of 139 nurses' staff that fulfilled the selecting criteria.

Published by Sciedu Press

\subsection{Inclusion criteria}

The study included registered nurses, male and female with different age and working experience more than three months at the cardiac care units and emergency departments and nurses agreed to participate in the study.

\subsection{Exclusion criteria}

The study excluded unregistered nurses, nurses with working experience less than three months at cardiac care units and emergency departments and nurses disagreed to participate.

\subsection{Instruments}

The tool used for data collection was standardized structured questionnaire. The questionnaire consisted of closed-ended questions which covered the following domain; the first section consisted of demographic data, gender, qualification, nursing experience and period of work in CCU and emergency department and second section consisted of knowledge information for initial drugs use to manage acute myocardial infarction (MONA), types of thrombolytic agents specifically streptokinase's characteristics, indications, relevant or absolute contraindications and complications; based on international guidelines for management of acute myocardial infarction. ${ }^{[17]}$ Most questions are multiple choice, each question item scoring from very poor to very good modified base on multiple Likert scale. ${ }^{[18]}$ The questionnaire reviewed by expert, information quality of internal consistency tested using Cronbach's alpha test, pre-tested in a ten subjects before the survey and any corrections were made. The volunteers received comprehensive training and explanation about details of questionnaire and they can fill it. ${ }^{[19]}$

\subsection{Data collection technique}

Data collected following direct interviewing technique for all participants use standardized structured questionnaire.

\subsection{Statistical data analysis}

Data obtained from study population cleaned, organized and analyzed used statistical computer software program version 19. An excel Microsoft program used for the graphs. ${ }^{[17]}$ Descriptive statistic applied (e.g. average, benchmark, frequency and percentage) and tests of significance performed $T$ test, chi square and correlation test. The chi square test applied to compare between some demographic variables such as training versus level of studied subjects' knowledge; used, $p$-value of $<.05$ considered statistically significant for the analysis. The analysis reflected the level of subjects knowledge based to Likert scale, ${ }^{[18]}$ which modified from very poor to very good score. 


\subsection{Ethical considerations}

Permission to carry out this study was granted by the ethics and research committee of the college of medical and health sciences at Elneelain University, agreement granted after written consents for leaders of health ministry of hospitals, $\mathrm{CCU}$ and emergency departments and verbal consent prior to interview obtained from participants.

\section{RESULT}

Table 1 showed five setting of the study, the studied subjects were nurses. Most of them were female (84.2\%) and their age ranged between $24-25$ years $(36.7 \%)$, with various qualifica- tions $66.9 \%$ had bachelor degree and $42.4 \%$ their experience in cardiac care units and emergency departments between 1-3 years. Also most of them didn't receive training only $21.6 \%$ received training of streptokinase, and $21.6 \%$ about advance life support, as well as $36.7 \%$ using of protocol and guidelines at CCU and ED.

Table 2 showed the average levels of nurses' knowledge appear in the table about initial drugs used for relieving the pain associated with acute myocardial infarction or to dissolve the clot into coronary artery during attack of acute myocardial infarction were significantly low compare with bench mark $(p$ value $=.000)$

Table 1. Background and professional characteristics of the study subjects

\begin{tabular}{|c|c|c|c|}
\hline Characteristics & & Frequency & Percentage (\%) \\
\hline \multirow{5}{*}{ Hospitals } & Khartoum & 41 & 29.5 \\
\hline & Omdurman & 35 & 25.2 \\
\hline & Elshaab & 28 & 20.1 \\
\hline & Ahmed Gassim & 20 & 14.4 \\
\hline & Bahri & 15 & 10.8 \\
\hline Total & & 139 & 100.0 \\
\hline \multirow{2}{*}{ Gender } & Male & 22 & 15.8 \\
\hline & Female & 117 & 84.2 \\
\hline Total & & 139 & 100.0 \\
\hline \multirow{5}{*}{ Age } & $<25$ & 49 & 35.3 \\
\hline & $25-29$ & 51 & 36.7 \\
\hline & $30-34$ & 15 & 10.8 \\
\hline & $35-39$ & 4 & 2.9 \\
\hline & $40+$ & 20 & 14.4 \\
\hline Total & & 139 & 100.0 \\
\hline \multirow{3}{*}{ Qualification } & Master & 14 & 10.1 \\
\hline & Bachelor (B.Sc) & 93 & 66.9 \\
\hline & Diploma & 32 & 23.0 \\
\hline Total & & 139 & 100.0 \\
\hline \multirow{3}{*}{ Experience years in CCU and ED } & $<1$ year & 50 & 36.0 \\
\hline & $1-3$ & 59 & 42.4 \\
\hline & $4+$ & 30 & 21.6 \\
\hline Total & & 139 & 100 \\
\hline \multirow{2}{*}{ Training of advance life support } & Yes & 30 & 21.6 \\
\hline & No & 109 & 78.4 \\
\hline Total & & 139 & 100 \\
\hline \multirow{2}{*}{ Training about streptokinase administration } & Yes & 21 & 15.1 \\
\hline & No & 118 & 84.9 \\
\hline Total & & 139 & 100 \\
\hline \multirow{2}{*}{ Work with Protocol and guidelines } & Yes & 51 & 36.7 \\
\hline & No & 88 & 63.3 \\
\hline Total & & 139 & 100.0 \\
\hline
\end{tabular}


Table 2. Average of participants' knowledge about an emergency drugs which use for initial management of an acute myocardial infarction $(\mathrm{n}=139)$

\begin{tabular}{lllllll}
\hline Items & Average & St Dev & Bench mark & $\boldsymbol{T}$-value & $\boldsymbol{p}$-Value & Conclusion \\
\hline Initial drugs given & 2.287 & 1.22 & 3 & 6.87 & .000 & Significant difference \\
Thrombolytic agents & 0.410 & 0.49 & 1 & 14.09 & .000 & Significant difference \\
Type of Thrombolytic & 0.942 & 0.961 & 3 & 25.23 & .000 & Significant difference \\
\hline
\end{tabular}

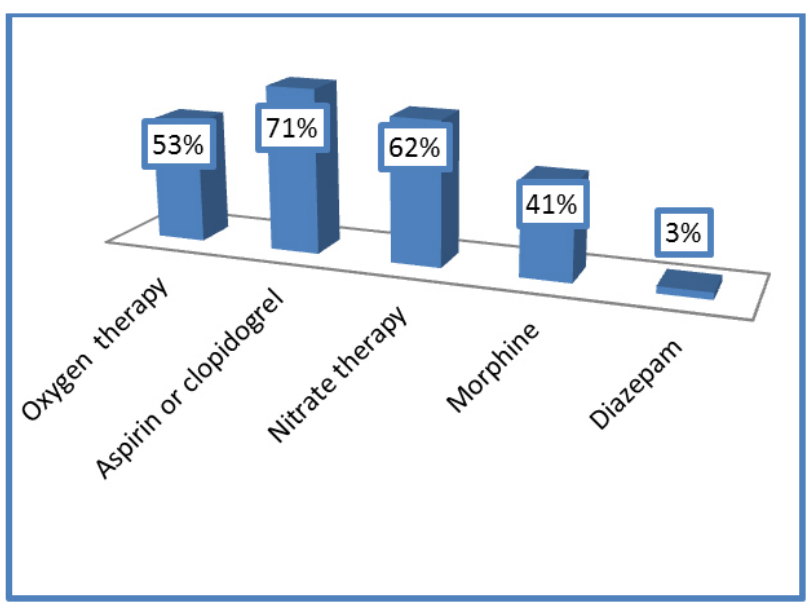

Figure 1. Participants' level of knowledge regard initial management which given to relief pain of acute myocardial infarction

Figure 1 reflected that levels of nurses knowledge about com- mon drugs use in managing the pain of acute myocardial infarction such as Aspirin, Nitrate and Oxygen were exceed the average level despite their knowledge about morphine was less than the average and very low knowledge related to diazepam used.

Figure 2 reflected that most of nurses had poor level of knowledge about thrombolytic agents which use to dissolve the clot inside the coronary artery, especially streptokinase's characteristics, indications, contraindications and complications.

Most levels of knowledge among trained nurses about streptokinase were poor and there were inverse correlation $(r)$. There were poor levels of knowledge about initial management for relieving pain, streptokinase' indications and relative contraindications among $38.1 \%, 76.2 \%$ and $66.7 \%$ of nurses correspondingly. In addition, there were very poor levels of knowledge among $33.3 \%$ of nurses regard complications of streptokinase.

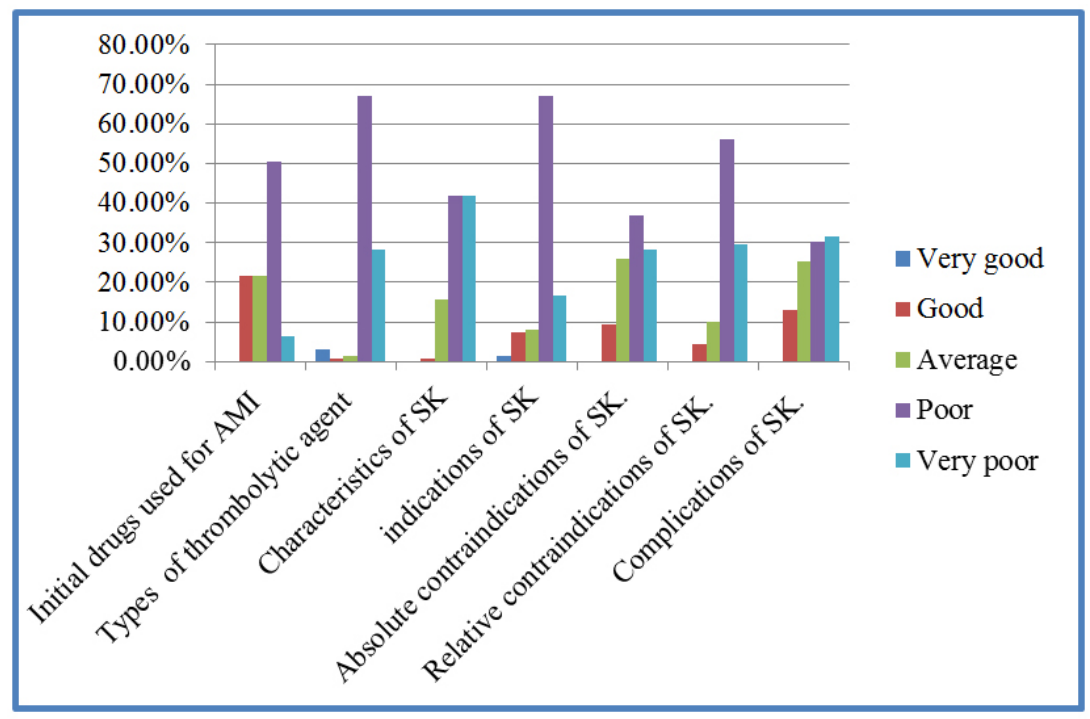

Figure 2. Participants' level of knowledge regard the type of the initial drugs and thrombolytic such as streptokinase $(\mathrm{n}=$ 139)

\section{Discussion}

The first goal for healthcare professionals in management of acute myocardial infarction is to diagnose the condition in a very rapid manner. ${ }^{[12]}$
The study delineated that most of studied subjects were female and most of them are youth with various qualifications. Most of them have bachelor degree and have experience of work 1-3 years in cardiac care units and emergency depart- 
ments. The numbers of nurses who received training or use myocardial infarction at areas of work are low (see Table 1). protocol and guidelines for management of patient with acute

Table 3. levels of knowledge of studied subjects regard initial drugs use to relive pain of myocardial infarction and streptokinase as specific drug versus training $(n=21)$

\begin{tabular}{|c|c|c|c|c|c|c|c|c|}
\hline \multirow{2}{*}{ Training } & \multicolumn{5}{|c|}{ Level of knowledge about streptokinase } & \multirow{2}{*}{ Total } & \multirow{2}{*}{$p$ value } & \multirow{2}{*}{$r$} \\
\hline & Very poor & Poor & Fair & Good & Very good & & & \\
\hline Initial drugs use to relive pain & $0.0 \%$ & $38.1 \%$ & $33.3 \%$ & $28.6 \%$ & $0.0 \%$ & $100.0 \%$ & .214 & -.160 \\
\hline Characteristics & $0.0 \%$ & $47.6 \%$ & $52.4 \%$ & $0.0 \%$ & $0.0 \%$ & $100.0 \%$ & .000 & -.438 \\
\hline Indications & $0.0 \%$ & $76.2 \%$ & $14.3 \%$ & $9.5 \%$ & $0.0 \%$ & $100.0 \%$ & . 183 & -.169 \\
\hline Absolute contraindications & $14.3 \%$ & $28.6 \%$ & $47.6 \%$ & $9.5 \%$ & $0.0 \%$ & $100.0 \%$ & .085 & -.174 \\
\hline Relative contraindications & $9.5 \%$ & $66.7 \%$ & $23.8 \%$ & $0.0 \%$ & $0.0 \%$ & $100.0 \%$ & .023 & -.186 \\
\hline Complications & $33.3 \%$ & $23.8 \%$ & $23.8 \%$ & $19.0 \%$ & $0.0 \%$ & $100.0 \%$ & .780 & -.030 \\
\hline
\end{tabular}

The study reflected that the average levels of nurses' knowledge about initial medications used for relieving pain or to dissolve the clot into coronary artery during attack of acute myocardial infarction was significantly low compare with bench mark ( $p$ value .000) (see Table 2). Specifically levels of nurses' knowledge about uses of aspirin, nitrate and oxygen exceeded the average. Despite their levels of knowledge about morphine and diazepam drugs was less than the average (see Figure 1). Also there was insignificant differences in their levels of knowledge between trained and nontrained nurses $p$ value (.214) and inverse correlation between levels of knowledge and training $(r=-.160)$ (see Table 3). This disagree with many studies that encouraging training, also there are many studies which supported and emphasized the important roles of the nurse during patients care according to specific protocol and guide lines through assessing the patient, providing the required care and teaching the patient. ${ }^{[20]}$ Morphine should be given IV to pain relief for patients with STEMI. Some forms of analgesia should be considered for patients with active chest discomfort. In the hospital for patients treated specifically with non-fibrinspecific thrombolytic (i.e., streptokinase), Creatinine level is provided $<3 \mathrm{mg} / \mathrm{dL}$. In patients with STEMI diagnosed in the out-of-hospital setting, reperfusion may be achieved by healthcare provider administration of fibrinolytic in the field. Alternatively fibrinolytic therapy may be administered on arrival at the hospital. If fibrinolysis is chosen as the reperfusion strategy, it should be started as soon as possible, ideally in the out-of-hospital setting, and should be administered by paramedics, nurses, or doctors under well-established protocols, competency training programs, and programs of continuous quality improvement with medical oversight. ${ }^{[21]}$ Training should aim to ensure that learners acquire and retain the skills and knowledge that will enable them to act correctly in an actual cardiac arrest. BLS and ALS knowl- edge and skills can be deteriorating as few as 3 to 6 months after training. Frequent assessments and, when needed, refresher training is recommended to maintain resuscitation knowledge and skills. ${ }^{[21]}$

Also this study reflects that most of nurses had poor level of knowledge about thrombolytic agents which use to dissolve the clot inside the coronary artery, especially streptokinase's characteristics, indications, contraindications and complications (see Figure 2). So most of trained nurses, who received training about streptokinase had poor levels of knowledge with insignificant difference related to non- trained one .Also there are inverse correlation $(r)$ between training and their levels of knowledge , this indicates low quality of training which they attended and absent of uses protocol and guideline for AMI management (see Table 3). This disagree with The Vienna STEMI registry result demonstrates that implementing the guidelines by forming an efficient network and implementing reperfusion therapy as quickly as possible after the onset of symptoms is beneficial in terms of reducing mortality and increasing the number of patients that receive reperfusion therapy. ${ }^{[19]}$

\section{Conclusion}

Most of the studied subjects had poor level of knowledge about initial management of acute myocardial infarction during an emergency situation with a significant inverse correlation between knowledge and training. This indicator of low quality training to whom they received.

\section{Unexpected findings}

The unexpected finding that the inverse relation between training and level of knowledge; this point lead to key interview with leaders of those emergency departments and $\mathrm{CCU}$ units about the quality of the training that nurses received. 


\section{Establish newness}

To establish a high quality program for continuous nurses training and periodically feedback.

\section{Explain discrepancies}

Disagree with the low level of knowledge for nursing staff who work at these sensitive areas.

\section{Further research and implications}

Plan for interventional studies to enhance nurses' competency and improve the performance of nursing practices.

\section{ACKNOWLEDGements}

I am grateful to the God who gave me the opportunity to complete this study. I wish to express my appreciation to the following persons: my parents, family for encouragement and support, my colleagues; for support with data collection, for all individuals who work at Elneelain University, especially staff of faculty of nursing science. Also acknowledge professor khlood Faik Salman from Duquesne University for her support and encouragement.

\section{CONFLICTS OF INTEREST DISCLOSURE}

The authors declare that there is no conflict of interest.

\section{REFERENCES}

[1] Elshazly M. Cardiovascular Emergencies. 2014. Available from: http://www.clevelandclinicmeded.com/medicalpubs/d iseasemanagement/cardiology/cardiovascular-emergen cies/

[2] NANDA book 12 Nursing Diagnosis for Myocardial Infarction. Available from: http://www. nanda-books.com/2012/10/12-nur sing-diagnosis-for-myocardial.html

[3] Lewis LL, et al. Medical Surgical Nursing, Assessment and Management of Clinical problems. 2015. Available from: https://www . am azon.com/Medical-Surgical-Nursing-2-Assessment-

[4] Bolooki HM, Askari A. Acute Myocardial Infarction. 2010. Available from: http://www.clevelandclinicmeded.com/m edicalpubs/diseasemanagement/cardiology/acute-myo cardial-infarction

[5] Grasso AW, Brener SJ. Complications of Acute Myocardial Infarction. 2014. Available from: http://www.clevelandclini cmeded.com/medicalpubs/diseasemanagement/cardiolog y/complications-of-acute-myocardial-infarction/

[6] Laham RJ, et al. Mechanical complications of acute myocardial infarction. 2016. Available from: http: //www . uptodate.com/contents/mechanical-complic ations-of-acute-myocardial-infarction

[7] World health organization (WHO). Coronary Heart Disease /selecting cause of death rate per 100.000. 2011. Available from: http://www.worldlifeexpectancy.com/cause-of-d eath/coronary-heart-disease/by-country

[8] Zafari AM, Yang EH. Myocardial Infarction Treatment \& Management. 2016. Available from: http://emedicine.medscape.com /article/155919-treatment

[9] Wikipedia. Acute coronary_syndrome last ST_segment_elevation infarction, Justification reference 71-79, last update. 2013. Available from: http://en.wikipedia.org/wiki/

[10] National Institute for Health and Clinical Excellence. Scottish Intercollegiate Guidelines Network, 2007, Acute coronary syndromes ISBN 1899893 741A. 2010. Available from: http://www.sign.a c.uk/pdf/sign93.pdf
[11] Figoros RN. Acute_cronary_Syndrome $/ 411204 / 1$ heart health center 2009. Available from: http://www. Heartdisease. About.com

[12] Zafari AM. Myocardial Infarction Treatment \& Manage. 2015. Available from: http://emedicine.medscape.com/article/1 55919-treatment

[13] Medicine Net-health. Thrombolytic agent definition. 2013. Available from: http://www.medicinenet.com/script/main/art. asp?articlekey $=5770$

[14] Ryan TJ. Initial Evaluation Management of Suspected Acute Coronary Syndrome in the Emergency Department, Boston University School of Medicine chest pain or discomfort. 2011.

[15] Hayman LL, et al. The role of nursing in promoting cardiovascular health Journal of the American College of Cardiology. 2015; 66(7): 864-866. Available from: http://content.onlinejacc.org/a rticle. aspx?articleid $=2423752$

[16] Jessica Heardman reviews a case study in which an ambulance crew managed the care of a patient later diagnosed with myocardial infarction Treating people with cardiac chest pain: role of paramedics. Available from: http://journals.rcni.com/doi/full/10.77 48/en2014.03.21.10.30.e635?mobileUi=0

[17] AHA. guidelines of emergency managements of patient with acute myocardial infarction. 2010. Available from: http://circ. ahajo urnals.org/

[18] The University of Melbourne. Stage program designed to assist $\mathrm{PhD}, \mathrm{ABN}: 84002705224$ Graduate Centre Australia. 2013. Available from: http://gradresearch.unimelb.edu.au/writin gcentre/

[19] Arntz HR, et al. Contemporary Management of Acute St segment Myocardial infarction, Thrombolysis and PCI as major treatment options. 2009.

[20] Abdelhameed AM, et al. Impact of a Designed Nursing Intervention protocol on Myocardial Infarction Patient's Outcome at a selected University Hospital in Egypt. Journal of Biology, Agriculture and Healthcare. 2013.

[21] Hazinski MF, et al. International Consensus on Cardiopulmonary Resuscitation and Emergency Cardiovascular Care Science With Treatment Recommendations. Circulation. 2010; 122: S250-S275. 